Pacific Journal of Mathematics

COTORSION THEORIES 


\title{
COTORSION THEORIES
}

\author{
STEPHEN D. BRONN
}

In this paper $A$ is a ring with unit, and $\operatorname{Mod}-A$ denotes the category of unitary right $A$-modules. The aim of the paper is to dualize the concept of torsion and develop the corresponding idea of cotorsion.

One generalization of torsion was given by Goldman, using what he called a kernel functor. These kernel functors are here dualized to give cokernel functors. Cokernel functors are categorized over $\operatorname{Mod}-A$.

The final section investigates what information the cotorsion functors can reveal about the homological properties of the rings under discussion.

1. Definition. An $I$-functor is a pair $(F, \lambda)$ where $F$ is an additive covariant functor from $\operatorname{Mod}-A$ to $\operatorname{Mod}-A$ and $\lambda$ is a natural transformation from the identity functor on $\operatorname{Mod}-A$ to $F$.

Thus if $M$ and $N$ are $A$-modules and $f \in \operatorname{Hom}_{A}(M, N)$ we have the commutative diagram

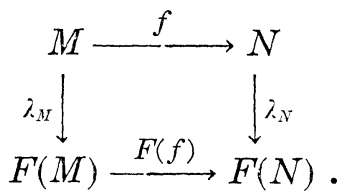

That is $\lambda_{N} f=F(f) \lambda_{M}$.

An $A$-module $M$ is said to be:

(i) $F$-reduced if $\lambda_{M}$ is a monomorphism.

(ii) $F$-divisible if $\lambda_{M}=0$.

(iii) $F$-cotorsion if $\lambda_{M}$ is an isomorphism.

(iv) $F$-d-strong if $D_{M}=$ cokernel of $\lambda_{M}$ is $F$-divisible.

In addition the $I$-functor $(F, \lambda)$ is said to be:

(a) epi if $\lambda_{M}$ is an epimorphism for every $M \in \operatorname{Mod}-A$.

(b) idempotent if $F(M)$ is $F$-cotorsion for every $M \in \operatorname{Mod}-A$.

(c) restricted idempotent if $F(M)$ is $F$-cotorsion whenever $M$ is $F$-reduced.

(d) $d$-strong if every $M \in \operatorname{Mod}-A$ is $F$ - $d$-strong.

The cotorsion completion functor of Matlis [4] is an example of a $d$-strong $I$-functor. This $I$-functor is idempotent if and only if the homological dimension of $Q$ ( $A$ is an integral domain and $Q$ is the quotient field of $A$ in this case) is one as an $A$-module.

If $A$ is a commutative ring and $S$ is a multiplicatively closed set of elements from $A$ then the localization of every module at $S$ is an 
$I$-functor. If every element of $S$ is a nonzero divisor this $I$-functor is idempotent and $d$-strong.

The following proposition follows directly from the definitions.

Proposition 1.1. Let $(F, \lambda)$ be an I-functor.

(a) Every F-cotorsion module is F-reduced.

(b) Every submodule of an F-reduced module is also F-reduced.

(c) Every quotient module of an F-divisible module is also $F$ divisible.

(d) $\operatorname{Hom}_{A}(M, N)=0$ whenever $M$ is F-divisible and $N$ is $F$ reduced.

(e) The additive condition is unnecessary if $(F, \lambda)$ is an epi I-functor or if $(F, \lambda)$ is idempotent and d-strong.

Proposition 1.2. Let $(F, \lambda)$ be an $I$-functor and $M$ be an $F$-dstrong $A$-module. For every $A$-module $N$ we denote by $\beta_{N}$ the group homorphism from $\operatorname{Hom}_{A}(F(M), N)$ to $\operatorname{Hom}_{A}(M, N)$ defined by composition with $\lambda_{Y}$.

(a) If $N$ is F-reduced $\beta_{N}$ is a monomorphism.

(b) If $N$ is F-cotorsion $\beta_{N}$ is an isomorphism.

Proof. (a) Suppose that $N$ is $F$-reduced and that $g$ is in the kernel of $\beta_{N}$. Thus $g \lambda_{M I}=0$. Let $u_{I I}: F(M) \rightarrow D_{M}$ be the cokernel of $\lambda_{M}$. There exists $h \in \operatorname{Hom}_{A}\left(D_{M}, N\right)$ such that $h u_{M}=g$. By 1.1

$$
\operatorname{Hom}_{A}\left(D_{M}, N\right)=0
$$

and therefore $h=0$. Hence $g=0$ and so $\beta_{N}$ is a monomorphism.

(b) By the preceding part we need only show that $\beta_{N}$ is onto if $N$ is $F$-cotorsion. Let $g \in \operatorname{Hom}_{A}(M, N)$, since $N$ is $F$-cotorsion $\lambda_{N}$ has an inverse $\lambda_{N}^{-1}$. Let $h=\lambda_{N}^{-1} F(g)$. Now $h \lambda_{M}=\lambda_{N}^{-1} F(g) \lambda_{M}=\lambda_{N}^{-1} \lambda_{N} g=g$ hence $\beta_{N}$ is onto.

Proposition 1.3. Let $J$ be a directed set and $B_{i}, i \in J$, be a family of $A$-modules indexed by $J$. Whenever $(F, \lambda)$ is an I-functor on Mod-A then:

(a) $\lim B_{i}$ is F-reduced if each $B_{i}, i \in J$, is F-reduced.

(b) $\lim B_{\imath}$ is F-divisible if each $B_{i}, i \in J$, is F-divisible.

(c) $\underset{i \in J}{\stackrel{i \in J}{\lim }} B_{i}$ is $F$-cotorsion if each $B_{i}, i \in J$, is F-cotorsion and if $(F, \lambda)$ is $d$-strong and restricted idempotent.

Proof. Let $M=\underset{j \in J}{\lim } B_{j}$ and $N=\underset{j \in J}{\lim } B_{\jmath}$ with respect to the defining 
homomorphisms $P_{i}: M \rightarrow B_{i}$ and $q_{i}: B_{i} \rightarrow N$ for $i \in J$.

(a) Suppose that each $B_{i}, i \in J$, is $F$-reduced. If $X \in \operatorname{Mod}-A$ and $h \in \operatorname{Hom}_{A}(X, M)$ such that $\lambda_{M} h=0$ then $0=F\left(P_{i}\right) \lambda_{M} h=\lambda_{B_{i}} P_{i} h$ for each $i \in J$. But $\lambda_{B_{i}}$ is a monomorphism thus $P_{i} h=0$ for each $i \in J$ and hence $h=0$ which means that $\lambda_{M}$ is a monomorphism.

(b) Suppose that each $B_{i}, i \in J$, is $F$-divisible. Now $\lambda_{N} q_{i}=$ $F\left(q_{i}\right) \lambda_{B_{i}}=0$ for each $i \in J$ and therefore $\lambda_{N}=0$.

(c) Suppose that each $B_{i}, i \in J$, is $F$-cotorsion and that $(F, \lambda)$ is $d$-strong and restricted idempotent. Thus $\lambda_{B_{i}}$ has an inverse $\lambda_{B_{i}}^{-1}$ for each $i \in J$ and so there exists $h \in \operatorname{Hom}_{A}(F(M), M)$ such that $P_{i} h=$ $\lambda_{B_{i}}^{-1} F\left(P_{i}\right)$ for each $i \in J$. Now $P_{i} h \lambda_{M}=\lambda_{B_{i}}^{-1} F\left(P_{i}\right) \lambda_{M}=\lambda_{B_{i}}^{-1} \lambda_{B_{i}} P_{i}=P_{i}$ for each $i \in J$ and thus $h \lambda_{M}=1_{M}$. By (a) $M$ is $F$-reduced and since $(F, \lambda)$ is restricted idempotent it follows that $F(M)$ is $F$-cotorsion and thus by $1.2 \beta_{F(M)}$ is an isomorphism. Since $\lambda_{M} h \lambda_{M}=\lambda_{M} 1_{M}=\lambda_{M}=1_{F(M)} \lambda_{M}$ it follows that $\lambda_{M} h=1_{F(M)}$ and thus $\lambda_{M}$ is an isomorphism and $M$ is $F$-cotorsion.

We now make a definition which allows us to compare $I$-functors.

Definition. If $(F, \lambda)$ and $(G, \alpha)$ are $I$-functors on Mod- $A$ and $\mu$ is a natural transformation from $F$ to $G$ such that $\mu \lambda=\alpha$ we say that $\mu$ is an $I$-morphism. If in addition $\mu_{M}$ is an isomorphism for each $M \in \operatorname{Mod}-A$ we say that $\mu$ is an $I$-isomorphism and that $(F, \lambda)$ and $(G, \alpha)$ are equivalent $I$-functors.

THEOREM 1.4. Let $(F, \lambda)$ and $(G, \alpha)$ be I-functors on Mod- $A$ where $(F, \lambda)$ is d-strong and $G(M)$ is $F$-cotorsion for every $M \in \operatorname{Mod}-A$. There exists an I-morphism $\mu$ from $(F, \lambda)$ to $(G, \alpha)$.

Proof. Let $M \in \operatorname{Mod}-A$, now $G(M)$ is $F$-cotorsion so by 1.2 there exists a unique $\mu_{M} \in \operatorname{Hom}_{A}(F(M), G(M))$ such that $\mu_{M} \lambda_{M}=\alpha_{M}$. Suppose now that $f \in \operatorname{Hom}_{A}(M, N)$. Thus $\mu_{N} F(f) \lambda_{M}=\mu_{N} \lambda_{N} f=\alpha_{N} f=G(f) \alpha_{M}=$ $G(f) \mu_{M} \lambda_{M}$. But $G(N)$ is $F$-cotorsion hence by $1.2 \mu_{N} F(f)=G(f) \mu_{M}$ which means that $\mu$ is an $I$-morphism.

2. The purpose of this section is to show that $F(A)$ is a ring for most $I$-functors $(F, \lambda)$.

THEOREM 2.1. Let $(F, \lambda)$ be an I-functor on Mod- $A$ such that $A$ is $F$-d-strong and $F(A)$ is $F$-cotorsion.

(a) $F(A)$ is a ring with unit and $\lambda_{A}$ is a ring homomorphism.

(b) Every F-cotorsion module $M \in \operatorname{Mod}-A$ is also a right $F(A)$ module.

(c) Whenever $M$ and $N$ are right $F(A)$-modules and $N$ is $F$ reduced as a right $A$-module then $\operatorname{Hom}_{A}(M, N)=\operatorname{Hom}_{F^{(A)}}(M, N)$.

(d) $F(A)$ is commutative if $A$ is commutative. 
Proof. Let $M$ be any $F$-cotorsion right $A$-module and let $x \in M$. Define $u_{x} \in \operatorname{Hom}_{A}(A, M)$ by $u_{x}(r)=x r$ for every $r \in A$. By 1.2 there exists $w_{x} \in \operatorname{Hom}_{A}(F(A), M)$ such that $w_{x} \lambda_{A}=u_{x}$.

(i) Clearly $u_{x}+u_{y}=u_{x+y}$ and so by $1.2 w_{x}+w_{y}=w_{x+y}$ for every $x, y \in M$.

(ii) Let $x \in M, s \in F(A)$ and set $y=w_{x}(s) . \quad w_{x} w_{s} \lambda_{A}(r)=w_{x}(s r)=$ $w_{x}(s) r=y r=u_{y}(r)=w_{y} \lambda_{A}(r)$ for every $r \in A$. Thus by $1.2 w_{x} w_{s}=w_{y}$.

Now $F(A)$ is $F$-cotorsion so by (i) and (ii) $F(A)$ becomes a ring under the multiplication $x y=w_{x}(y)$ where $x, y \in F(A)$. By the same taken $M$ is a right $F(A)$-module.

If $r, s \in A$ let $x=\lambda_{A}(r)$, then $\lambda_{A}(r s)=\lambda_{A}(r) s=x s=u_{x}(s)=w_{x} \lambda_{A}(s)=$ $\lambda_{A}(r) \lambda_{A}(s)$ and therefore $\lambda_{A}$ is a ring homomorphism. Clearly $\lambda_{A}(1)$ is the unit of $F(A)$.

Suppose now that $M$ and $N$ are right $F(A)$-modules and that $N$ is $F$-reduced when considered as a right $A$-module. Let $f \in \operatorname{Hom}_{A}(M$, $N)$ and $x \in M$. Define $h, g \in \operatorname{Hom}_{A}(F(A), N)$ by $g(s)=f(x) s$ and $h(s)=f(x s)$ for $s \in F(A)$. It is easily seen that $g \lambda_{A}=h \lambda_{A}$ so by 1.2 $g=h$. That is $f$ is a right $F(A)$-module homomorphism and so $\operatorname{Hom}_{A}(M, N)=\operatorname{Hom}_{F(A)}(M, N)$.

Now assume that $A$ is commutative. Let $r \in A, x \in F(A)$ and set $y=\lambda_{A}(r)$. Define $g \in \operatorname{Hom}_{A}(F(A))$ by $g(s)=s \lambda_{A}(r)=s y=w_{s}(y)=$ $u_{s}(r)=s r$ for $s \in F(A)$. Now $g \lambda_{A}=w_{y} \lambda_{A}$ and so by $1.2 g=w_{y}$ and therefore $\lambda_{A}(r) s=s \lambda_{A}(r)$ for every $r \in A, s \in F(A)$.

Define $h_{x} \in \operatorname{Hom}_{A}(F(A), F(A))$ by $h_{x}(s)=s x-x s$ where $x \in F(A)$. Now $h_{x} \lambda_{A}=0$ by the previous paragraph and so by $1.2 h_{x}=0$ which means that $F(A)$ is commutative. This completes the proof of the theorem.

Definition. Let $(F, \lambda)$ be an $I$-functor such that $A$ is $F$-d-strong and $F(A)$ is $F$-cotorsion. By $2.1 F(A)$ is a ring with unit $\lambda_{A}(1)$ where 1 is the unit of $A$. We define a new $I$-functor $(\bar{F}, \bar{\lambda})$ on $\operatorname{Mod}-A$ by $F(M)=M \otimes_{A} F(A)$ for every $M \in \operatorname{Mod}-A$ and $\bar{\lambda}(y)=y \otimes \lambda_{A}(1)$ for every $y \in M$.

THEOREM 2.2. Let $(F, \lambda)$ be an idempotent, $d$-strong I-functor on Mod-A. $(F, \lambda)$ and $(\bar{F}, \bar{\lambda})$ are equivalent I-functors on Mod- $A$ if and only if $\bar{F}(M)$ is $F$-cotorsion for every module $M \in \operatorname{Mod}-A$.

Proof. If $(F, \lambda)$ and $(\bar{F}, \bar{\lambda})$ are equivalent $I$-functors then $F(M)$ and $\bar{F}(M)$ are isomorphic for every $M \in \operatorname{Mod}-A$. But $F(M)$ is $F$ cotorsion and thus $\bar{F}(M)$ is $F$-cotorsion.

Conversely suppose that $\bar{F}(M)$ is $F$-cotorsion for every $M \in \operatorname{Mod}-A$. By 1.4 there exists an $I$-morphism $\mu$ from $(F, \lambda)$ to $(\bar{F}, \bar{\lambda})$. By 2.1 $F(M)$ is a right $F(A)$-module for every module $M \in \operatorname{Mod}-A$. Thus 
there exists $\alpha_{M} \in \operatorname{Hom}_{A}(\bar{F}(M), F(M))$ such that $\alpha_{M}(y \otimes s)=\lambda_{M}(y) s$ for every $y \in M, s \in F(A)$. Now $\alpha_{M} \mu_{M} \lambda_{M}=\lambda_{M}$ and $F(M)$ is $F$-cotorsion thus by $1.2 \alpha_{M} \mu_{M}=1_{F(M)}$ for every $M \in \operatorname{Mod}-A$.

Let $y \in M$ and $s \in F(A) \mu_{M} \alpha_{M}(y \otimes s)=\mu_{M}\left(\lambda_{M}(y) s=\mu_{M}\left(\lambda_{M}(y)\right) s\right.$ by 2.1 thus $\mu_{M} \alpha_{M}=1_{\bar{F}(M)}$ and hence $\mu$ is an $I$-isomorphism.

3. In this section the kernel functor of Goldman [3] is dualized. Stenstrom (6) studied a particular type of this kernel functor in one attempt to extend the work of Matlis [4].

Definition. A cokernel functor on $\operatorname{Mod}-A$ is an epi $I$-functor $(F, \lambda)$ on Mod- $A$ such that whenever $g \in \operatorname{Hom}_{A}(M, N)$ is an epimorphism then the following diagram is a pushout

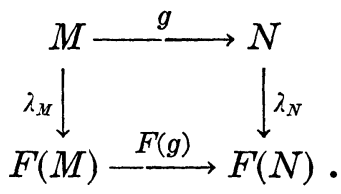

Proposition 3.1. Every cokernel functor is idempotent and $d$ strong.

Proof. Let $(F, \lambda)$ be a cokernel functor on $\operatorname{Mod}-A .(F, \lambda)$ is clearly $d$-strong since it is an epi $I$-functor. Suppose that $M \in \operatorname{Mod}-A$ and $N=F(M)$. Now $F\left(\lambda_{M}\right) \lambda_{M}=\lambda_{N} \lambda_{M}$ thus $F\left(\lambda_{M}\right)=\lambda_{N}$ since $\lambda_{M}$ is an epimorphism. This means that

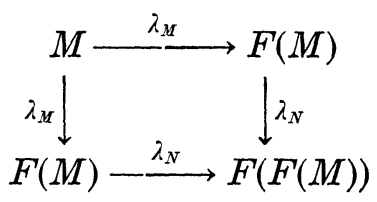

is a pushout and therefore $\lambda_{N}$ is an isomorphism. Hence $(F, \lambda)$ is idempotent.

Proposition 3.2. Let $(F, \lambda)$ be an epi I-functor on Mod-A. The following statements are equivalent:

(i) $(F, \lambda)$ is a cokernel functor.

(ii) $F$ is a right exact functor.

(iii) $(F, \lambda)$ is idempotent and any homomorphic image of an $F$ cotorsion module is also $F$-cotorsion.

Proof. The equivalence of (i) and (ii) follows from Mitchell [5] Chapter 1, Proposition 13.2*.

Suppose that $(F, \lambda)$ is a cokernel functor. By $3.1(F, \lambda)$ is idem- 
potent. If $g \in \operatorname{Hom}_{A}(M, N)$ is an epimorphism and $M$ is $F$-cotorsion then

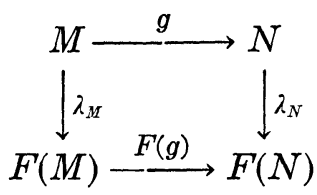

is a pushout where $\lambda_{M}$ is an isomorphism. Thus by Mitchell [5] Chapter 1, Propositions $7.2^{*}$ and $20.2^{*} \lambda_{N}$ is an isomorphism. This shows that (i) implies (iii).

Conversely assume (iii). Let $g \in \operatorname{Hom}_{A}(M, N)$ be an epimorphism and let $u: G \rightarrow M$ be the kernel of $g$. Let $v: F(M) \rightarrow X$ be the cokernel of $\lambda_{M} u$. Since $F(M)$ is $F$-cotorsion it follows that $X$ is also $F$-cotorsion. Since $g$ is the cokernel of $u$ there exists $h \in \operatorname{Hom}_{A}(N, X)$ such that $h g=v \lambda_{M}$. Thus by 1.2 there exists $f \in \operatorname{Hom}_{A}(F(N), X)$ such that $f \lambda_{N}=h$. Therefore, $f F(g)=v$ and so $F(g): F(M) \rightarrow F(N)$ is the cokernel of $\lambda_{M} u$. Hence by Mitchell [5] Chapter 1, Proposition 13.2*

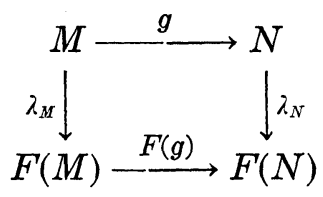

is a pushout and so $(F, \lambda)$ is a cokernel functor.

THEOREM 3.3. If $(F, \lambda)$ is a cokernel functor on Mod- $A$ then $(F, \lambda)$ and $(\bar{F}, \bar{\lambda})$ are equivalent I-functors.

Proof. Let $J=$ kernel of $\lambda_{A}$. By 3.1 and $2.1 F(A)$ is a ring and $J$ is a 2-sided ideal of $A$. Also $F(A)$ is ring isomorphic to $A / J$.

Let $M$ be any free right $F(A)$-module. $\quad M$ can be embedded in a direct product of copies of $F(A)$. By 1.3 a direct product of copies of $F(A)$ is $F$-cotorsion and so by $1.1 M$ is $F$-reduced. But $\lambda_{M}$ is an epimorphism thus $M$ is $F$-cotorsion.

If $N$ is any right $F(A)$-module then $N$ is the homomorphic image of a free $F(A)$-module $M$ and so by $3.2 N$ is $F$-cotorsion. If $U \in \mathrm{Mod}$ $A$ then $\bar{F}(U)$ is a right $F(A)$-module and so $\bar{F}(U)$ is $F$-cotorsion. Thus by $2.2(F, \lambda)$ and $(\bar{F}, \bar{\lambda})$ are equivalent $I$-functors.

If $J$ is any 2-sided ideal of $A$ then $M \rightarrow M \otimes_{A} A / J$ is easily seen to define a cokernel functor on Mod- $A$. Combining this with 3.3 we have a complete classification of all cokernel functors.

4. We now investigate the relationship between homological 
properties of $F(A)$ and those of $A$ where $(F, \lambda)$ is an $I$-functor on Mod- $A$, much in the same manner as Turnidge [7].

LEMma 4.1. Let $(F, \lambda)$ be a restricted idempotent, $d$-strong $I$ functor such that $A$ is $F$-reduced. If every F-reduced right $A$-module is flat then $A$ is left semi-hereditary.

Proof. Every direct product of $F$-reduced modules is $F$-reduced by 1.3 and submodules of $F$-reduced modules are also $F$-reduced by 1.1. Thus every torsionless right $A$-module is $F$-reduced since $A$ is $F$-reduced and therefore every torsionless right $A$-module is flat. Hence by [2, Thm. 4.1] $A$ is left semi-hereditary.

We will need to refer to a restricted idempotent, $d$-strong $I$-functor where $A$ is $F$-reduced frequently throughout this section. We therefore call such an $I$-functor special for easy reference.

Lemma 4.2. Suppose that $(F, \lambda)$ is a special I-functor on Mod-A. Every $F$-reduced right $A$-module is also $\bar{F}$-reduced.

Proof. Let $M \in \operatorname{Mod}-A$ be $F$-reduced. Since $(F, \lambda)$ is restricted idempotent, $F(M)$ is $F$-cotorsion and hence by 2.1 is a right $F(A)$ module. Thus there exists $u_{M} \in \operatorname{Hom}_{A}(\bar{F}(M), F(M))$ such that $u_{M}(y \otimes r)=$ $\lambda_{M}(y) r$ for every $y \in M r \in F(A)$. That is $u_{M} \bar{\lambda}_{M}=\lambda_{M}$ and since $\lambda_{M}$ is a monomorphism so is $\bar{\lambda}_{M}$. Therefore $M$ is $\bar{F}$-reduced.

The following theorem investigates the weak dimension (WD) of $\bar{F}$-reduced modules if the global weak dimensions (GWD) of $F(A)$ and $A$ are known.

Theorem 4.3. Let $(F, \lambda)$ be a special I-functor on Mod-A such that $F(A)$ is flat as a right $A$-module. If GWD $F(A) \leqq m$ and GWD $A \leqq$ $n+1$ where $m$ and $n$ are nonnegative integers such that $m \leqq n$ then WD $M \leqq n$ for every $\bar{F}$-reduced right $A$-module $M$.

Proof. Let $M \in$ Mod- $A$ be $\bar{F}$-reduced. Since GWD $F(A) \leqq m$ thus $\bar{F}(M)=M \otimes_{A} F(A)$ has weak dimension $\leqq m$ as an $F(A)$-module. Hence by [1, Prop. VI 4.12] $M \otimes_{A} F(A)$ has weak dimension $\leqq m$ as an $A$-module.

Let $B=$ cokernel $\bar{\lambda}_{M}: M \rightarrow M \otimes_{A} F(A)$. This gives rise to exact sequences

$$
\begin{aligned}
& \operatorname{Tor}_{k+1}^{A}(B, X) \longrightarrow \operatorname{Tor}_{k}^{A}(M, X) \longrightarrow \operatorname{Tor}_{k}^{A}\left(M \bigotimes_{A} F(A), X\right) \longrightarrow \\
& \quad \operatorname{Tor}_{k}^{A}(B, X)
\end{aligned}
$$

for every nonnegative integer $k$ and left $A$-module $X$. If $k>n$ then $k+1>n+1$ and $k>m$. Thus $\operatorname{Tor}_{k+1}^{A}(B, X)=0=\operatorname{Tor}_{k}^{A}\left(M \bigotimes_{A} F(A)\right.$, 
$X)$ so $\operatorname{Tor}_{k}^{A}(M, X)=0$ and therefore WD $M \leqq n$.

CoRollary 4.4. Let $(F, \lambda)$ be a special I-functor on Mod- $A$ such that $F(A)$ is flat as a right A-module and GWD $F(A)=0$. The following statements are equivalent:

(i) $A$ is left semi-hereditary.

(ii) GWD $A \leqq 1$.

(iii) Every F-reduced right A-module is flat.

(iv) Every $\bar{F}$-reduced right $A$-module is flat.

Proof. (i) $\Rightarrow$ (ii) follows from [1, Prop. VI 2.9]

(ii) $\Rightarrow$ (iv) is a consequence of 4.3 .

(iv) $\Rightarrow$ (iii) is immediate from 4.2 .

(iii) $\Rightarrow$ (i) is immediate from 4.1 .

Theorem 4.5. Let $(F, \lambda)$ be a special I-functor on Mod-A. If $F(A)$ is projective as a right $A$-module and is a semi-simple Artinian ring, the following statements are equivalent:

(i) $A$ is right hereditary.

(ii) $M$ is projective for every $F$-reduced $M \in \operatorname{Mod}-A$.

Proof. Since $(F, \lambda)$ is special every right ideal of $A$ is $F$-reduced by 1.1. Thus (ii) $\Rightarrow$ (i) is immediate.

(i) $\Rightarrow$ (ii). Let $M \in \operatorname{Mod}-A$ be $F$-reduced. By $4.2 M$ is $\bar{F}$-reduced so we have an exact sequence

$$
0 \longrightarrow M \longrightarrow M \otimes_{A} F(A) \longrightarrow B \longrightarrow 0 \text {. }
$$

Now $F(A)$ is semi-simple Artinian so $M \otimes_{A} F(A)$ is a projective $F(A)$-module. $F(A)$ is a projective $A$-module and thus $M \otimes_{A} F(A)$ is a projective $A$-module. Therefore, by [1, I Thm. 5.4] $M$ is a projective $A$-module.

We now investigate a relationship between the global dimension (GD) of $F(A)$ and the injective dimension (ID) of $F$-cotorsion modules over a commutative ring.

THEOREM 4.6. Let $A$ be a commutative ring and $(F, \lambda)$ a special $I$ functor on Mod- $A$ such that $F(A)$ is flat as an $A$-module. If $\operatorname{GD} F(A) \leqq$ $n$ where $n$ is a nonnegative integer then ID $M \leqq n$ for every $F$-cotorsion $M \in \operatorname{Mod}-A$. In addition if GWD $F(A) \leqq m$ where $m$ is a nonnegative integer then WD $M \leqq m$ for every $F$-cotorsion $M \in \operatorname{Mod}-A$.

Proof. Let $M \in$ Mod- $A$ be $F$-cotorsion. By $2.1 M$ is an $F(A)$ module and by [1, Prop. VI 4.1.3 and 4.1.2] we have isomorphisms $\operatorname{Ext}_{F(A)}^{k}\left(X \otimes_{A} F(A), M\right) \cong \operatorname{Ext}_{A}^{k}(X, M)$ and $\operatorname{Tor}_{k}^{A}(M, X) \cong \operatorname{Tor}_{k}^{F^{\prime}(A)}(M$, 
$X \bigotimes_{A} F(A)$ ) for every $X \in \operatorname{Mod}-A$. Since GD $F(A) \leqq n$ and GWD $F(A) \leqq$ $m$ it follows that ID $M \leqq n$ and WD $M \leqq m$.

Corollary 4.7. Let $A$ be a commutative ring and $(F, \lambda)$ a special I-functor on Mod-A such that $F(A)$ is flat as an $A$-module and such that $A / I$ is $F$-cotorsion for every nonzero ideal $I$ of $A$. Then GWD $A \leqq$ GWD $F(A)$.

Proof. Assume GWD $F(A)=m$. Then by $4.6 \mathrm{WD} A / I \leqq m$ for every ideal $I$ of $A$. Hence GWD $A \leqq m$.

An example of a special $I$-functor of the type in the preceding corollary is the cotorsion completion functor of Matlis [4] which is given by $M \rightarrow \operatorname{Ext}_{A}^{1}(K, M)$ for every $M \in \operatorname{Mod}-A$ where $A$ is an integral domain and $K=Q / A$ where $Q$ is the quotient field of $A$.

\section{REFERENCES}

1. H. Cartan and S. Eilenberg, Homological Algebra, Princeton University Press, Princeton, N. J., 1956.

2. S. U. Chase, Direct products of modules, Trans. Amer. Math. Soc., 97 (1960), 457473.

3. O. Goldman, Rings and modules of quotients, J. Algebra, 13 (1969), 10-47.

4. E. Matlis, Cotorsion Modules, Memoirs of the A.M.S., No. 49 (1964).

5. B. Mitchell, Theory of Categories, Academic Press, New York, 1965.

6. B. Stenstrom, On the completion of modules in an additive topology, J. Algebra, $\mathbf{1 6}$ (1970), 523-540.

7. D. R. Turnidge, Torsion theories and semi-hereditary rings, Proc. Amer. Math. Soc., 24 (1970), 137-143.

Received July 6, 1972. 



\section{PACIFIC JOURNAL OF MATHEMATICS}

\section{EDITORS}

RICHARD ARENS (Managing Editor)

University of California

Los Angeles, California 90024

\author{
R. A. Beaumont \\ University of Washington \\ Seattle, Washington 98105
}

J. Dugundu*

Department of Mathematics

University of Southern California

Los Angeles, California 90007

D. Gilbarg and J. Milgram

Stanford University

Stanford, California 94305

\section{ASSOCIATE EDITORS}
E. F. BECKENBACH
B. H. NeumanN
F. WOLF
K. YosHIDA

\section{SUPPORTING INSTITUTIONS}

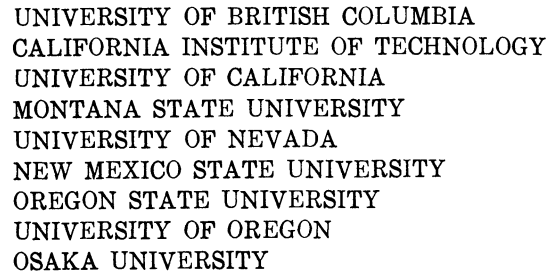

UNIVERSITY OF BRITISH COLUMBIA CALIFORNIA INSTITUTE OF TECHNOLOGY

UNIVERSITY OF CALIFORNIA

MONTANA STATE UNIVERSITY

UNIVERSITY OF NEVADA

NEW MEXICO STATE UNIVERSITY

OREGON STATE UNIVERSITY

UNIVERSITY OF OREGON

OSAKA UNIVERSITY

\author{
UNIVERSITY OF SOUTHERN CALIFORNIA \\ STANFORD UNIVERSITY \\ UNIVERSITY OF TOKYO \\ UNIVERSITY OF UTAH \\ WASHINGTON STATE UNIVERSITY \\ UNIVERSITY OF WASHINGTON \\ * * * \\ AMERICAN MATHEMATICAL SOCIETY \\ NAVAL WEAPONS CENTER
}

The Supporting Institutions listed above contribute to the cost of publication of this Journal, but they are not owners or publishers and have no responsibility for its content or policies.

Mathematical papers intended for publication in the Pacific Journal of Mathematics should be in typed form or offset-reproduced, (not dittoed), double spaced with large margins. Underline Greek letters in red, German in green, and script in blue. The first paragraph or two must be capable of being used separately as a synopsis of the entire paper. Items of the bibliography should not be cited there unless absolutely necessary, in which case they must be identified by author and Journal, rather than by item number. Manuscripts, in duplicate if possible, may be sent to any one of the four editors. Please classify according to the scheme of Math. Rev. Index to Vol. 39. All other communications to the editors should be addressed to the managing editor, or Elaine Barth, University of California, Los Angeles, California, 90024.

50 reprints are provided free for each article; additional copies may be obtained at cost in multiples of 50 .

The Pacific Journal of Mathematics is issued monthly as of January 1966. Regular subscription rate: $\$ 48.00$ a year (6 Vols., 12 issues). Special rate: $\$ 24.00$ a year to individual members of supporting institutions.

Subscriptions, orders for back numbers, and changes of address should be sent to Pacific Journal of Mathematics, 103 Highland Boulevard, Berkeley, California, 94708.

PUBLISHED BY PACIFIC JOURNAL OF MATHEMATICS, A NON-PROFIT CORPORATION

Printed at Kokusai Bunken Insatsusha (International Academic Printing Co., Ltd.), 270, 3-chome Totsuka-cho. Shinjuku-ku, Tokyo 160, Japan.

* C. R. DePrima California Institute of Technology, Pasadena, CA 91109, will replace J. Dugundji until August 1974.

Copyright (C) 1973 by

Pacific Journal of Mathematics

All Rights Reserved 


\section{Pacific Journal of Mathematics}

Vol. 48, No. $2 \quad$ April, 1973

Mir Maswood Ali, Content of the frustum of a simplex................

Mieczyslaw Altman, Contractors, approximate identities and factorization

in Banach algebras ................................ 323

Charles Francis Amelin, A numerical range for two linear operators ...... 335

John Robert Baxter and Rafael Van Severen Chacon, Nonlinear functionals

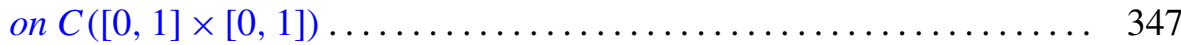

Stephen Dale Bronn, Cotorsion theories....................... 355

Peter A. Fowler, Capacity theory in Banach spaces............... 365

Jerome A. Goldstein, Groups of isometries on Orlicz spaces ........... 387

Kenneth R. Goodearl, Idealizers and nonsingular rings . ............ 395

Robert L. Griess, Jr., Automorphisms of extra special groups and

nonvanishing degree 2 cohomology ..................... 403

Paul M. Krajkiewicz, The Picard theorem for multianalytic functions . . . . 423

Peter A. McCoy, Value distribution of linear combinations of axisymmetric harmonic polynomials and their derivatives ...................

A. P. Morse and Donald Chesley Pfaff, Separative relations for

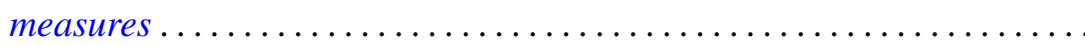

Albert David Polimeni, Groups in which $\operatorname{Aut}(G)$ is transitive on the

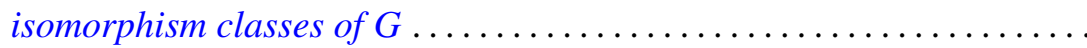

Aribindi Satyanarayan Rao, Matrix summability of a class of derived

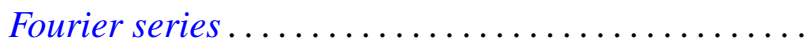

Thomas Jay Sanders, Shape groups and products

Ruth Silverman, Decomposition of plane convex sets. II. Sets associated

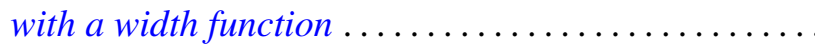

Richard Snay, Decompositions of $E^{3}$ into points and countably many flexible dendrites.............................

John Griggs Thompson, Nonsolvable finite groups all of whose local subgroups are solvable, IV ...

Robert E. Waterman, Invariant subspaces, similarity and isometric equivalence of certain commuting operators in $L_{p} \ldots$

James Chin-Sze Wong, An ergodic property of locally compact amenable

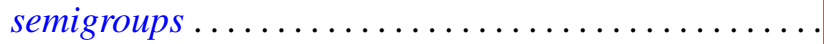

Julius Martin Zelmanowitz, Orders in simple Artinian rings are strongly equivalent to matrix rings ....................... 